\title{
Spatial navigation based on novelty mediated autobiographical memory
}

\author{
Emilia I. Barakova and Tino Lourens \\ Brain Science Institute RIKEN, 2-1 Hirosawa, Wako-shi, Saitama, 351-0198, Japan. E- \\ mail:emilia@brain.riken.jp. \\ Honda Research Institute Japan Co., Ltd. 8-1 Honcho, Wako-shi, Saitama, 351-0114, \\ Japan.E-mail: tino@jp.hondari.com.
}

\begin{abstract}
This paper presents a method for spatial navigation performed mainly on past experiences. The past experiences are remembered in their temporal context, i.e. as episodes of events. The learned episodes form an active autobiography that determines the future navigation behaviour. The episodic and autobiographical memories are modelled to resemble the memory formation process that takes place in the rat hippocampus. The method implies naturally inferential reasoning in the robotic framework that may make it more flexible for navigation in unseen environments. The relation between novelty and life-long exploratory (latent) learning is shown to be important and therefore is incorporated into the learning process. As a result, active autobiography formation depends on latent learning while individual trials might be reward driven. The experimental results show that learning mediated by novelty provides a flexible and efficient way to encode spatial information in its contextual relatedness and directionality. Therefore, performing a novel task is fast but solution is not optimal. In addition, learning becomes naturally a continuous process - encoding and retrieval phase have the same underlying mechanism, and thus do not need to be separated. Therefore, building a "life long" autobiography is feasible.
\end{abstract}

\section{Introduction}

Referring to the memories of experienced events is a usual way to orient in novel situations. Common feature of an embodied agent (that could be animal, human or robot) is that it continuously gathers information about the surrounding world through experiencing sequences of events. Such subjectively experienced sequences are encoded by episodic and autobiographical memory systems in living organisms [1].

Tulving and Markowitsch [2] at present divide memory into five systems. The most complex memory system is the episodic-autobiographical one which requires self-conscious reflection and is embedded in the dimensions of time and locus. Semantic memory, on the other hand, is context-free fact memory. On the more automatic, implicit level, perceptual memory, procedural memory and the priming system constitute the other three long-term memory systems with perceptual memory allowing the assessment of novelty/familiarity of perceptual stimuli, procedural memory referring largely to sensory-motor skills and simple conditioning and priming to an enhanced identification of objects.

Many models in computer science and robotics exploit the characteristics of the 
semantic (factual) memory. However, memory for events and their relatedness is the way higher organisms build their knowledge. Many spatial navigation tasks in robotics are inspired by navigation behavior of animals. For instance, insects behavior have been simulated in [3][4][5][6]. While insects navigation has mainly reactive nature, the behavior of mammals is memory-driven [7][8][9][10][11]. We aim at memory determined behavior that relies on the neural mechanisms underlying episode formation. Episodic information encoding is related to the hippocampal modelling and with this respect our work is most closely related to [7][8][9][10][11]. We understand episodic memory as including event information within its temporal relatedness and directionality, as modelled in theoretical studies. Moreover, our aim is to obtain behaviors that are mainly driven by old experiences. The experiences are encoded during exploratory learning, a process guided by environmental novelty.

Novelty is a known factor that gates learning in natural and artificial systems $[31][13][14][15][17][18]$. The relation between novelty and behaviour has received much attention by experimental neuroscientists [12][13][14][15][16][17][18], but there is not enough experimental evidence to build a good computational model. The hippocampus is a brain structure where episodic and perceptual information come together, and where environmental novelty is signalled. Therefore we develop a novelty method that uses the available experimental evidence from hippocampal functioning and optimized it for robotics implementation. Novelty detection is related to experienced episodes rather than a novel place in the environment, as in other robotic studies [19] [20].

There is a robotic study that introduces an autobiographical agent as an embodied agent which dynamically reconstructs its individual history (autobiography) during its lifetime [21]. However, they do not consider the neural correlates of autobiographical memory as MTL or prefrontal cortex. Instead, an algebraic model that is not related to the brain processes underlying the autobiographical encoding is used.

This paper is structured as follows: Section 2 proposes a hypothesis based on experimental studies; The computational framework is developed in Section 3. Some results are shown in Section 4. A discussion of the state of the research and its perspectives is made in Section 5.

\section{Motivation and hypothesis}

The involvement of the hippocampus in episodic memory encoding has been elaborated extensively (see for instance [23]). This encoding is associated with area CA3 of the hippocampus, where due to vast recurrent connectivity the past information is represented in the current representations.

Although the importance of the hippocampus for episodic memory function is undisputed, there is a considerable debate regarding the precise role of this brain region. The hippocampus is reported to be involved in encoding and retrieval of event information, including item and relational information. It is differentially more involved in memory for relational information than in memory for item information.

Recent works report that the hippocampus is selectively engaged in detecting novel ties in the environment. Tulving and Markowitsch [1] argue that perceptual memory allows the assessment of novelty/familiarity of perceptual stimuli. Therefore, our interest 
is in CA1 area of the hippocampus, to which projections of sensory-bound stimuli come together with episodes of recent memories as formed in area CA3 [25][26]. The same sensory-bound pattern is transferred through both: the direct and the indirect pathway from the enthorinal cortex (EC) to CA1 area, as illustrated in Fig. 1.

Our computational model follows the general structure and functioning of rat hyppocampus. We make the following hypothesis: If perceptual memory is involved in novelty detection of places, and the hippocampus is involved in episodic memory formation, the relational information that comes from $\mathrm{CA} 3$ to $\mathrm{CA} 1$ area interferes with the perceptual information to account for novelty of remembered episodes, rather of novelty of particular places, for which perceptual memory alone is indicative.

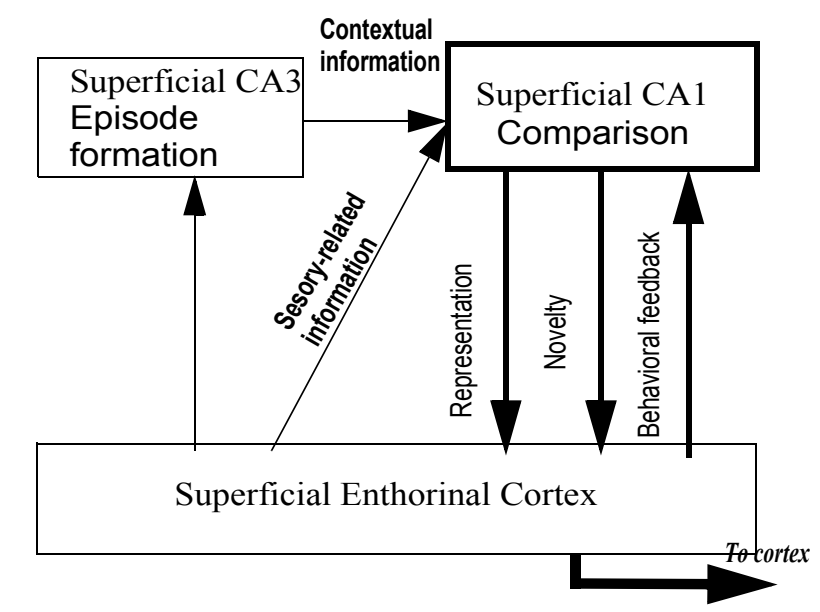

Fig. 1. Computational scheme based on the assumed functionality of the hippocampal formation, accentuating on the comparative role of the CA1 area.

CA1-3 denote resemblance with areas 1-3 of cornu ammonis within the hippocampus. The sensory bound and episodic memory related representations are compared to indicate the familiarity. The areas are denoted as Superficial CA1,

$\mathrm{CA} 3$ or EC to indicate that modelling is not biologically precise.

\section{Computational model inspired by the hippocampal paradigm.}

The proposed model aims at novelty driven encoding and recall that facilitates inferential reuse of old memories. Its global functioning is illustrated in Fig. 1. Three structures, resembling EC, CA1 and CA3 areas form the representation, that is further used for navigation. The computations are performed in the superficial CA1 and CA3 areas. The final representation is formed in CA1 area, that is activated through two pathways one directly from $\mathrm{EC}$ area and one through the indirect activation pathway going from EC through CA3 areas. The pattern that reaches the CA1 area via the direct pathway is 
organized on pattern similarity, not on topological principle. Since the same projected pattern from the $\mathrm{EC}$ area reaches within a small time interval areas $\mathrm{CA} 1$ and $\mathrm{CA} 3$, the connection between the current most active neurons in these two areas is strengthened also. This automatically activates the complete episode to which the pattern in CA3 area corresponds, and therefore the contextual information from this episode is transferred to area CA1.

The activation of the individual patterns in the superficial $\mathrm{EC}$ area is derived by the established theory [24] that the cells in the rat hippocampus fire when the rat is at particular location of the environment. Because of this feature, these cells are also called place cells. If the rat moves through the environment, at every particular place a number of place cells fire. Cells that code for places in nearest vicinity fire most strongly, while the cells that fire for more distant location fire less. The activity of the place cells can be modelled by a Gaussian for the open environments, where place cells show non-directional firing. Therefore, the movement of a simulated rat at every place of the environment is characterized by a particular pattern of firing, containing the active place cells in its vicinity. The level of activity of every place cell depends on the distance between the rat position and the place fields centres. The mathematical description of this process has been shown in several works before, here we show the outcome of a simulation of two activation patterns formed by simulation of place cells and rat route (Fig. 2). These patterns are external-world related and are further transmitted through the direct pathway.

Activity pattern, coming through the indirect pathway, represents the episodic influence to the representation in CA1 area. It is formed within a network structured as a two layer lattice of neurons, corresponding to the EC and CA3 layers. There are two types of synoptic plasticity, that take place within this network. Between the layers, the afferent connections from superficial EC to the superficial CA3 area are trained through a modified Hebbian rule:

$$
\Delta w_{i j}{ }^{C A-E C}=\alpha_{1} g\left(E C_{i} C A_{j}-w_{i j}{ }^{C A-E C} C A_{j}^{2}\right),
$$

where $\alpha_{1}$ is learning rate, notation $C A-E C$ shows the starting and destination layer of the connection (coming from $\mathrm{EC}$, reaching CA layer) the indices $i$ and $j$ denote neurons on the input and output layer, correspondingly. The CA layer is not denoted as CA1 or $\mathrm{CA} 3$, because the learning rule is used for EC-CA1 as well as EC-CA3 learning. The term $-w_{i j}{ }^{C A-E C} C A^{2}{ }_{j}$ is needed due to internal instability of the Hebbian rule.

The lateral connections within CA3 area are two types: lateral inhibitory and lateral connections that provide the temporal context. The lateral inhibitory connections, denoted as LI have a sharpening effect on the transmitted to CA3 area activations. Eq.(3).

$$
\Delta w_{j}{ }^{L I-C A}=\alpha_{2} g\left(L \dot{I} \cdot C A_{j}-\alpha_{3}{ }^{w_{i}}{ }^{L I-C A} C A_{j}^{2}\right)
$$

where $\alpha_{2}, \alpha_{3}$ are learning rates, $g$ is a gating factor. 


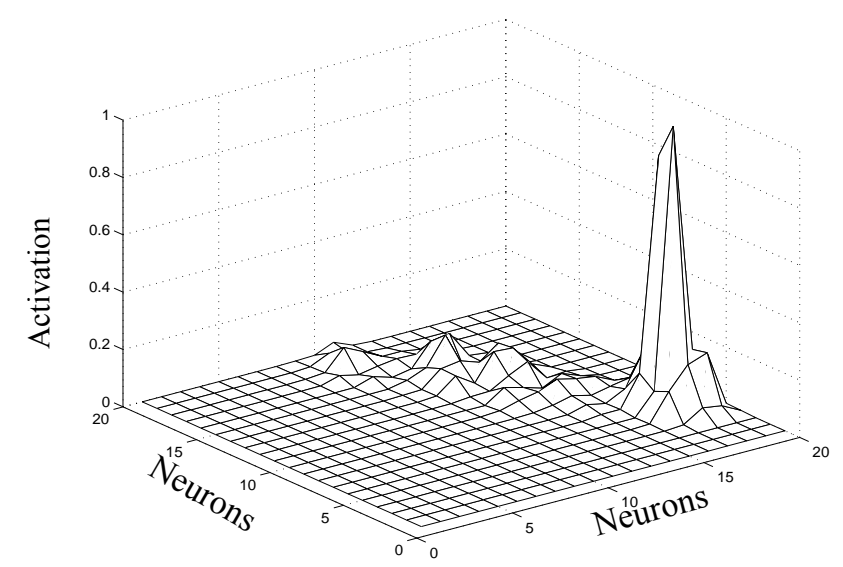

Fig. 2. A learned episode in CA3 area. Activation of a single pattern induces activation within the whole episode.

By far, the representation made within the layer denoted as CA3 has not the intrinsic capacity for temporal association. This quality is obtained by applying a hebbian rule with asymmetric time window over the neurons within the CA3 lattice, since in CA3 layer lateral connections exist between the neurons. Note, that this learning is not affected by the lateral inhibitory connections, denoted as LI in Eq. 3. It concerns the learning due to the exitatory lateral connections only. The asymmetric time window has been simulated to correspond to the experimental measurements as found by [27], see also [28]. The lateral excitatory learning rule is:

\section{bound}

$$
\Delta v_{i j}(t)=\sum_{\tau=0} \operatorname{LTP}(\tau) C A_{i}(t) C A_{j}(t-\tau)+\operatorname{LTD}(-\tau) C A_{i}(t-\tau) C A_{j}(t)
$$

where LTP and LTD denotes long term potentiation/ long term depression, respectively, as found by [27][28] and adapted into an asymmetrical time window function. $T_{e p}$ shows the length of the temporal interval, considered in episode formation. By introducing the temporal aspect, learning in CA3 is episodic. Therefore, when a single pattern in the learned episode is activated the whole episode is activated. Figure 4 shows this effect for a simplified connectivity within CA3 layer. Individual neurons are connected to their immediate neighbors in the simplification needed for visualization process.

The learning in the direct pathway EC-CA1 has the same learning dynamics as described in equations (1) and (2). However, the learning outcome differs substantially, because of the different connectivity between the EC-CA3 and EC-CA1 layers. In the first case, the topological connections are predominant, while in the second, the areas are fully connected. Therefore, the EC-CA1 learning does not preserve the topology of the pattern activation. 
CA3-CA1 synaptic plasticity has the following dynamics. If $\mathrm{k}$ and $\mathrm{l}$ are neurons from CA3 and CA1 areas respectively, the connection between them is strengthened if both of them are simultaneously active and weakened if the activation of one decreases. A term that regulates the unbounded growth of the weights is added (Eq. 4). Note that the activation of CA3 neurons might not indicate single neuron activation but the influence of an active episode.

$$
\Delta c_{l k}{ }^{C A 3-C A 1}=\theta\left(C A 3_{l} C A 1_{k}-c_{l k}{ }^{C A 1-C A 3} C A 1^{2}{ }_{k}\right)
$$

The sensory bound representation of the direct pathway, and the episodic representation of the indirect pathway, come together in CA1 area, where the comparison (novelty/familiarity judgements) takes place. Note, that at the same time, the CA1 area gets input from the current pattern of EC area and a pattern from CA3 area, which has not been included in an episode yet.

For robotics learning task, several simplifications of the biologically plausible learning algorithm are made. The place cell formation process was replaced by recordings with an omni-directional distance sensor, since the two patterns look similar and uniquely represent a position in space. Fig. 3 . shows couples of similar patterns. The patterns shown above are obtained by simulating the place cell formation by an exploration process, while the plots below are recorded by an omnidirectional distance sensor.

The simplification in the learning process are derived from the observation, that topology preserving learning between the EC and CA3 layer does not have substantial contribution to the learning outcome. In contrast, the self-organizing process between $\mathrm{EC}$ and $\mathrm{CA} 1$, and the temporal association learning within the CA3 layer are essential.

Therefore, the learning process ignores the plasticity of $\mathrm{EC}$ to $\mathrm{CA} 3$ connections and the inhibitory lateral connections. The hebbian learning rule between the layers followed by lateral inhibition is replaced by a modification of the competitive hebbian learning algorithm [8][16], adapted for processing of sequences.

\section{Active autobiography through exploration.}

The mammals, who are able to form episodic memories, and especially humans can remember a particular experience for the whole life span. This fact suggests, that episodic memory encoding is an efficient process, i.e. together with the encoding a decision of the content and importance of the encoded information is taken.

Autobiographical memory overlaps with the concept of episodic memory. It is a memory of one's personal past, but concerns a longer time span. With this respect an analogy can be made with the robotics paradigm of life-long learning. This paper investigates autobiography formed by remembered episodes only. We call it active autobiography to accentuate on its dynamic character. 
a)

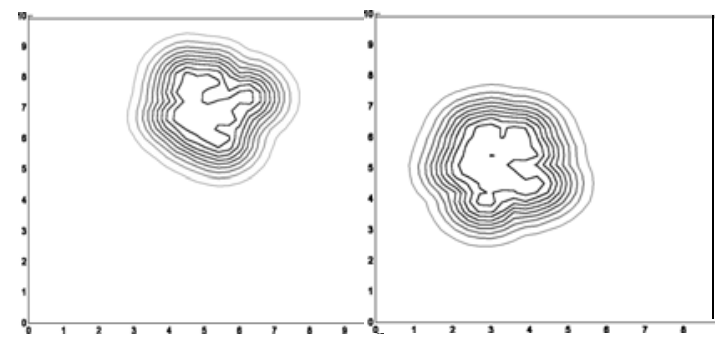

b)

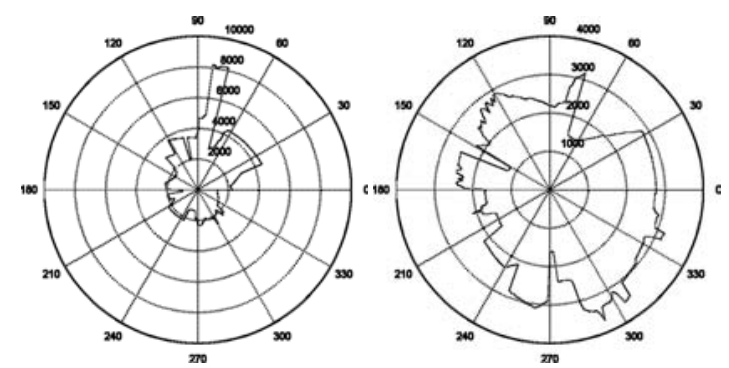

Fig. 3. Samples of sensory patterns. a) Simulation of the place cells formation process. b) Record by omnidirectional range sensor of a robot.

For building an active autobiography, a latent learning scenario is used. Latent learning is an association of indifferent stimuli or situations with one another without immediate reward. The phenomenon is clearly exemplified in exploratory behavior, and is also known as exploratory learning [24][29][30]. In robotics research the term exploratory learning is preferred, while latent learning is mostly used in animal studies. To compare the results, however, target locations are marked.

To illustrate the active autobiography formation, the episodic encoding when the robot reaches a sample location are shown in Fig.4a). The learning outcome is shown in Fig. 4b). It indicates, that trajectories (episodes) that are considered similar are not remembered.

Furthermore, the goal reaching by free exploration is shown. To connect the neural representation with the goal reaching navigation behavior, an algorithm we showed in [31] is used. Once a position in the environment is recognized, the robot behavior is guided by the remembered episode. This behavior is illustrated in Fig. 4c. Initially, starting from the beginning of the coordinate system, the reactive behavior is shown. The non-smooth part of the trajectory shows navigation guided by the remembered episode. 
8.

a)

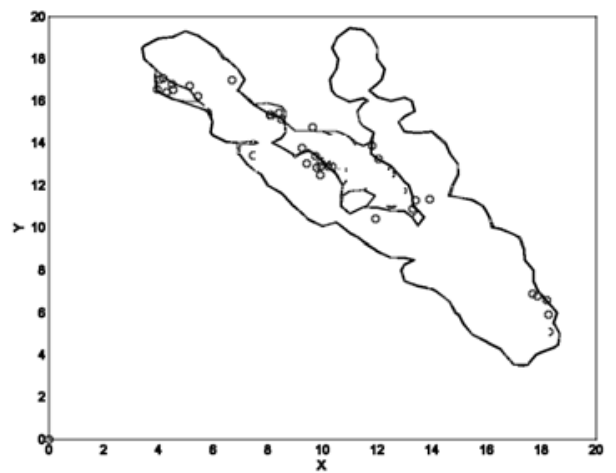

b)

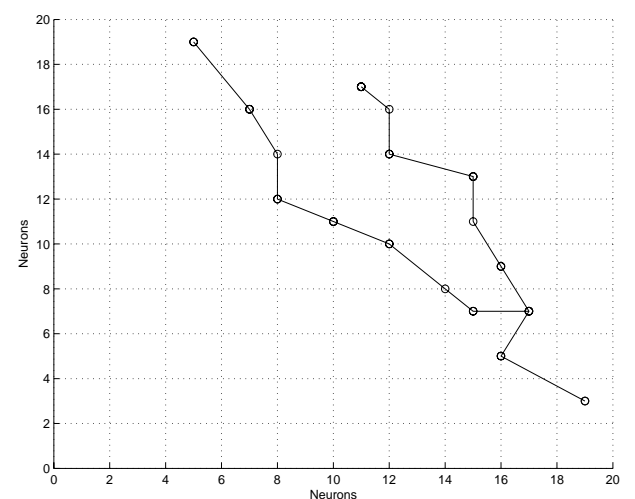

c)

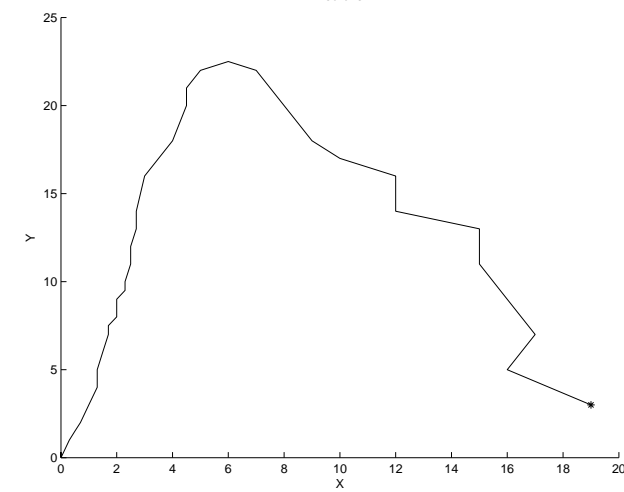

Fig. 4. a) 3 experienced episodes. b) Representation of the encoded episodes.

c) Reaching a target location by encountering a known path. 


\section{DISCUSSION}

This study shows spatial navigation that is enhanced by robots memories. Formation of the remembered episodes is mediated by novelty/familiarity discrimination method based on the hippocampal modelling for robotics exploration. The embodied nature of an animal and robot makes this parallel useful, and the functional efficiency of the hippocampal encoding, while performing both tasks: episodic encoding and novelty detection, suggests an optimal computational scheme.

The impact of novelty is two-fold: it allows an efficient encoding in the exploration phase and it is a basis for flexible reuse of memories in the recall phase.The same computational paradigm is used in both cases, which makes possible on-line implementation. The paper accentuates on the methodological part and shows simulations of episodic memory encoding and navigation that uses the remembered episodes. A comparison of the method with exploration that uses actor critic learning is made and the advantages of our method for larger environment is obvious. However, the results need good explanation of the method and why this comparison is plausible. Because of that they will be shown elsewhere. The navigation trajectories become more efficient with enriching the autobiography of the stored exploration episodes, since the optimal trajectory is recorded.

\section{References}

[1] Tulving, E., Donaldson, W.: Organization of memory. New York: Academic Press, 1972.

[2] Tulving, E., Markowitsch, H.J. . Episodic and declarative memory: Role of the hippocampus. Hippocampus, 8, 198-204, (1998).

[3] Svennebring, J., Koenig, S.:Building Terrain-Covering Ant Robots: A Feasibility Study,Autonomous Robots 16 (3): 313-332, May 2004

[4] Leerink, L., Schultz S.Jabri M.: A Reinforcement Learning Exploration Strategy based on Ant Foraging Mechanisms. Proceedings of the Sixth Australian Conference on Neural Networks, Sydney, Australia, (1995).

[5] Lambrinos, D., Muller R., Labhart T., Pfeifer R., Wehner R.: A mobile robot employing insect strategies for navigation. Robotics and Autonomous Systems, 30:39-64 (2000).

[6] Wehner, R., Michel, B., Antonsen, P.: Visual navigation in insects: coupling of egocentric and geocentric information ,The Journal of Experimental Biology 199, 129-140 (1996).

[7] Arleo, A., Gerstner, W.: Spatial cognition and neuro-mimetic navigation: A model of hippocampal place cell activity. Biological Cybernetics, 83, 287-299 (2000).

[8] Balakrishnan, K., Bousquet, O., Honavar, V.: Spatial learning and localization in rodents: A computational model of the hippocampus and its implications for mobile robots. Adaptive Behavior, $\underline{7}, 173-216$ (1999).

[9] Prescott, T. J.: Spatial representation for navigation in animats. Adaptive Behavior, 4, 85123 (1996).

[10] Scholkopf, B., Mallot, H. A.: View-Based Cognitive Mapping and Path Planning. Adaptive Behavior Vol.3, No.3, 311-348, (1995). 
[11] Trullier, O. \& Meyer, J.-A.: Animat navigation using a cognitive graph. In R. Pfeifer, B. Blumberg, J.-A. Meyer, \& S. W. Wilson (Eds.), From Animals to Animats 5 (pp. 213-222). Cambridge, MA: MIT Press (1998).

[12] Kemp, A., Manahan-Vaughan, D.: Hippocampal long-term depression and long-term potentiation encode different aspects of novelty acquisition. Proc Natl Acad Sci U S A. May 25;101(21):8192-7 (2004).

[13] Knight, R., Nakada, T.: Cortico-limbic circuits and novelty: a review of EEG and blood flow data. Rev. Neurosci. 9, 57-70 (1998).

[14] Lisman, J.E., Otmakova, N.: Storage, recall, and novelty detection of sequences by the hippocampus: elaborating on the SOCRATIC model to account for normal and aberrant effects of dopamine. Hippocampus 11, 551-558 (2001).

[15] Li S, Cullen WK, Anwyl R, Rowan MJ.: Dopamine-dependent facilitation of LTP induction in CA1 by exposure to spatial novelty. Nat Neurosci. 6(5):526-31 (2003).

[16] Moses, S., Sutherland, R., McDonald, R.: Differential involvement of amygdala and hippocampus in responding to novel objects and contexts. Brain Res. Bull. 58, 517 (2002).

[17] Bevins, RA, Bardo, MT.: Conditioned increase in place preference by access to novel objects: antagonism by MK-801.”Behav Brain Res 1999;99:53-60.

[18] Galani, R, Weiss I, Cassel J-C, Kelche C.: Spatial memory, habituation, and reactions to spatial and nonspatial changes in rats with selective lesions of the hippocampus, the entorhinal cortex or the subiculum. Behav Brain Res 1998; 96:1 12 (1998).

[19] Barakova, E., Zimmer, U.: Dynamic Situation and Trajectory Discrimination by Means of Clustering and Summation of Raw Range Data", AISTA, Australia, (2000).

[20] Marsland S.: Novelty Detection in Learning Systems", Neural Comp. Surveys, 3: (2003).

[21] Dautenhahn, K., C. Nehaniv: Artificial Life and Natural Stories, Proc. of the Third International Symposium on Artificial Life and Robotics (AROB III), pp. 435-439, (1998).

[22] Barakova E. and Lourens T., "Prediction of Rapidly Changing Environmental Dynamics for Real Time behavior Adaptation using Visual Information”, Workshop on Dynamic Perception, Bochum, Germany, Nov. 2002.

[23] Bunsey, M. and Eichenbaum, H. B.: Conservation of hippocampal memory function in rats and humans. Nature (London), 379 (6562), 255-257 (1996).

[24] O'Keefe, J., Nadel, L.: The Hippocampus as a Cognitive Map, Clarendon Press, Oxford (1978).

[25] Jensen O and J.E. Lisman, "Hippocampal CA3 region predicts memory sequences: accounting for the phase precession of place cells." Learning and Memory 3:279-287.

[26] O'Reilly, R.C. and McClelland, J.L., "Hippocampal conjunctive encoding, storage, and recall: avoiding a trade-off”. Hippocampus, 1994, 4(6):661-682.

[27] Zhang L., Tao H., Holt C., Harris W. Poo M. A critical window for cooperation and competition among developing retinotectal synapses. Nature 395:37-44 1998.

[28] Markram, H., Lubke, H., Frotscher, M., and Sakmann, B., Regulation of synaptic efficacy by coincidence of postsynaptic aps and epsps. Science, 275:213-215, 1997.

[29] Manning, A.: An Introduction to Animal Behaviour, Edward Arnold, London (1979).

[30] Renner, M.J.: Learning during exploration: The role of behavioral topography during exploration in determining subsequent adaptive behavior, Int. J. Comp. Psychol. 2, 43(1988).

[31] Barakova, E.I.: Emergent behaviors based on episodic encoding and famili|arity driven retrieval, Lecture Notes in Artificial Intelligence, vol.3192, pp. 109-118, Springer-Verlag (2004). 